Gut, 1976, 17, 1007-1011

\title{
Evidence for the mixing of residue in the human gut
}

\author{
H. S. WIGGINS AND J. H. CUMMINGS ${ }^{1}$ \\ From the Medical Research Council Gastroenterology Unit, Central Middlesex Hospital, London
}

SUMMARY Mixing of residue in the gut has been studied in 24 normal subjects using five different types of radio-opaque pellets. By giving single doses of different pellets to the subjects on three successive days and measuring their appearances in the stool by radiographs evidence of mixing was found in $83 \%$ of studies. Mean transit time (MTT) of markers through the gut was measured from the single dose studies (MTT-S) and also by giving the pellets to subjects continuously with each meal (MTT-C). By comparing MTT-C with MTT-S evidence was found for a mixing pool with exponential decay. The turnover time of this pool was estimated as about $30 \%$ of the total MTT.

It was an early observation that markers fed on successive days to healthy people tended to 'catch-up' or overlap with those given previously (Alvarez and Freedlander, 1924; Hoelzel, 1930). Mixing of intestinal contents from one day with that of the next must therefore occur in the gut. The nature of this mixing affects the interpretation of transit time measurements, particularly estimation of the maximum time that a compound may remain in the gut. This is relevant to the hypothesis which postulates the production of potential carcinogens in the bowel by an extensive series of chemical changes (Hill et al., 1971).

We have therefore assessed the extent and significance of mixing in the gut by giving different radio-opaque markers on successive days.

\section{Methods}

Twenty-four healthy male subjects aged 22-32 years took part in 84 studies. The protocol was fully explained to each subject and had been approved by the Ethical Committee of the Central Middlesex Hospital.

\section{MARKERS}

Five different radio-opaque markers were used: (1) Large cubes (Radio-opaque pellets, Portex Ltd.), (mean weight $31 \mathrm{mg}$, specific gravity (SG) 1.25);

'Present address: Dunn Nutrition Laboratory, Milton Road, Cambridge.

Received for publication 7 July 1976
(2) small cubes (mean weight $7 \cdot 8 \mathrm{mg}$, SG $1 \cdot 25$ );

(3) small circles (mean weight $8 \mathrm{mg}$, SG 1.63);

(4) large circles (mean weight $16 \mathrm{mg}$, SG 1.63);

(5) rods (mean weight $4.8 \mathrm{mg}$, SG 1.71 ). In a previously reported study, where details of their manufacture and characteristics are reported (Cummings et al., 1976), no significant difference in mean transit time between the various markers was found when 20 of each type were given simultaneously on 10 occasions to a group of subjects. Type 5 (rods) were made by cutting $3 \mathrm{~mm}$ lengths from radioopaque PVC tubing, external diameter $1.5 \mathrm{~mm}$ (Portex R1). This marker was used only in the large dose studies.

\section{PROCEDURE}

On 71 occasions in 22 subjects a dose of 20 markers was taken with breakfast on three successive days, a different marker being used each day. In 39 of these three-dose studies mean transit time was being measured simultaneously using a continuous marker method (MTT-C). In this method five radioopaque markers are taken with each meal (15 per day) over periods of several weeks; faecal marker output is measured by radiographs of the stools and MTT-C is then calculated from the number of markers retained in the gut (Cummings et al., 1976). In addition, on eight occasions, subjects who were taking the continuous marker for measurement of MTT-C took a single dose of 20 markers with breakfast. Four subjects also took a single dose of $\mathbf{4 0 0}$ type 5 markers with breakfast but not while taking any other marker. 

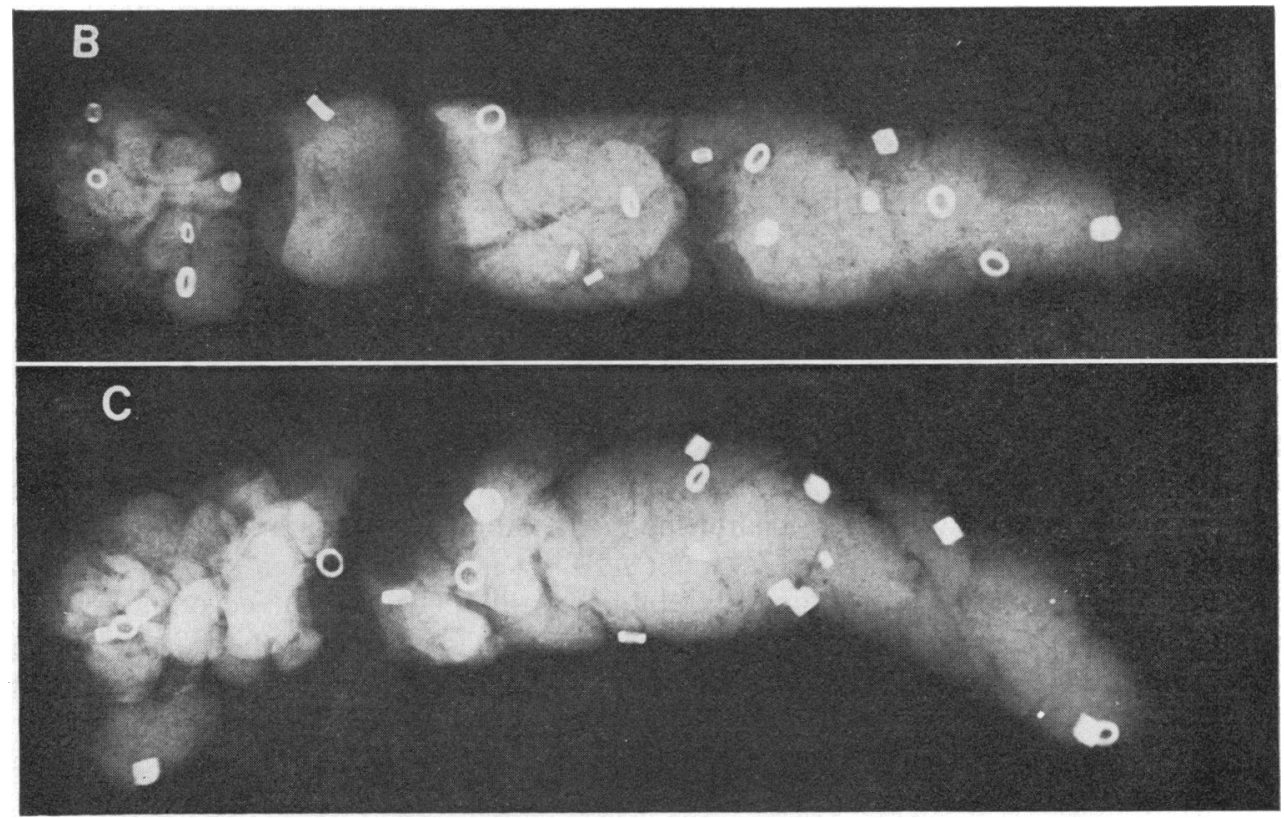

Fig. 1 Radiographs of two stools passed on day $5(B)$ and day $6(C)$ by a subject who on day 1 took 20 small circles, day 2, 20 large circles, day 3, 20 large cubes, and day 4, 20 small cubes, each as a single dose with breakfast.

In all these studies stools were collected from the time the first marker was taken until all had been recovered. Each bowel movement was collected separately, the time noted, and then stored until radiographs were taken. For Fig. 1 the stools were collected in such a way as to enable identification of the sequence in which the stool was passed by collecting it onto a slowly moving cardboard sheet.

Mean transit time (MTT) This is the average time taken for marker to pass through a system.

MTT-C Where the marker is given continuously to the subject the MTT for the marker is the same as the turnover time (Zilversmit, 1960). In the present case it is calculated by dividing the average number of markers retained in the gut over a 24-hour period by 15 (the number ingested daily).

Normalisation of transit times In order to get an overall view of the pattern of marker excretion in this group of subjects the transit time of individual markers has been normalised to make a composite picture possible. One hundred and twenty-five single doses of marker were given to the subjects while transit was simultaneously being measured by the continuous marker method. Transit of individual markers from the single doses has been expresssed as a proportion of the MTT-C, the value used for
MTT-C being the average for the days during which the single dose of marker passed through the gut (Figs. 2 and 3).

\section{Results}

All the subjects had a normal bowel habit and were eating a variety of diets. During the study their average daily faecal weight was $106 \pm 48 \mathrm{~g}$ (SD) and the mean interval between stools $27 \cdot 1 \pm 15 \cdot 8 \mathrm{~h}$. The average MTT was $58 \cdot 7 \pm 21.0 \mathrm{~h}$.

Mixing of markers in the gut has been characterised in two main ways.

\section{OCCURRENCE OF MIXED MARKERS IN STOOL} In the presence of a daily bowel action, as occurred in the majority of these subjects, the presence in one stool of all three types of marker is strongly suggestive of mixing. This occurred in 59 out of 71 studies $(83 \%)$ (Table). This situation could have occurred without mixing if bowel habit was less than daily. Unequivocal evidence of mixing is provided when, after the subject has taken a dose of different markers on three successive days, two or more stools contain all three types of marker given. Twenty-four out of $71(34 \%)$ studies showed unequivocal evidence of mixing by this criterion. Mixing of markers in individual stools is demonstrated in Fig. 1. 


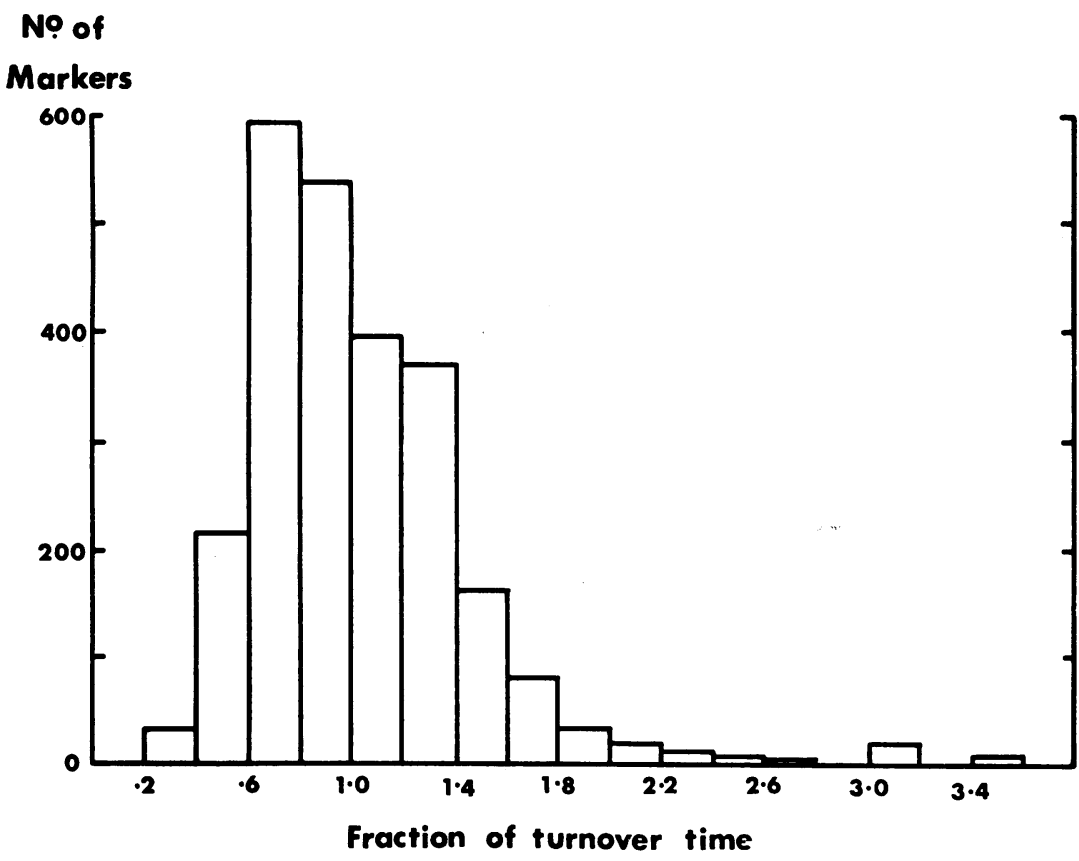

Fig. 2 Distribution of transit times of markers given as a single dose expressed as a fraction of the turnover time (determined by the continuous administration of markers). Each column represents the total number of markers for which the transit time was a certain fraction of the turnover time.

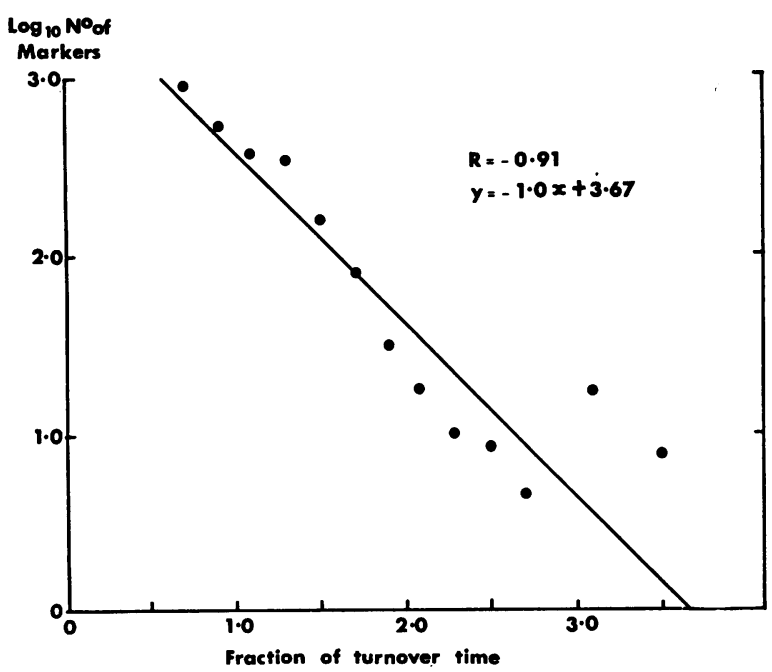

Table Frequency of occurrence of mixed markers in individual stools

Number of studies in which.

\begin{tabular}{|c|c|c|c|c|c|c|c|c|}
\hline$x=$ & 0 & $I$ & 2 & 3 & 4 & 5 & 6 & \\
\hline $\begin{array}{l}\text { Only } 1 \text { type of marker was } \\
\text { present in } x \text { stools }\end{array}$ & 10 & 16 & 27 & 11 & 4 & 1 & 2 & 71 \\
\hline $\begin{array}{l}2 \text { types of marker were } \\
\text { present in } x \text { stools }\end{array}$ & 13 & 25 & 21 & 4 & 7 & 0 & 1 & 71 \\
\hline $\begin{array}{l}\text { All } 3 \text { types of marker were } \\
\text { present in } x \text { stools }\end{array}$ & 12 & 35 & 19 & 4 & 0 & 1 & 0 & 71 \\
\hline
\end{tabular}

also taking continuous markers for the measurement of MTT-C. The distribution is not a normal one. The modal value lies between $0 \cdot 6-0 \cdot 8$, while mean is 1.0. In Fig. 3 the decline of the curve is plotted logarithmically. This approximates closely to a straight line suggesting an exponential component to the excretion of the markers.

When a dose of 20 markers is given each marker

Fig. 3 The logarithmic plot of the distribution of marker transit time shown in Fig. 2.

2. DISTRIBUTION OF TRANSIT TIMES OF INDIVIDUAL MARKERS GIVEN AS A SINGLE DOSE Figure 2 shows the distribution of transit times for the individual markers passed expressed as a fraction of the MTT-C. It is derived from the 125 single doses of marker given to the 23 subjects who were 
MTT (range 2-2-4.3) compared with $2 \cdot 0$ times for the 20 marker doses.

\section{Discussion}

The human gut is essentially an unbranched tube and so it would be reasonable to expect that residue would pass through in a sequential manner. This is not supported by the results from the present studies which indicate that significant mixing of dietary residue from one day with the next occurs.

One explanation for the mixing of different markers could be that they have different transit times. However, we have previously shown that these markers have similar transit times (Cummings et al., 1976). Furthermore, Findlay et al. (1974) have shown that markers as dissimilar as chromium sesquioxide and polyethylene glycol (Carbowax 4000) behave identically in normal subjects, although they dissociate in some pathological conditions.

Demonstration of mixing is difficult. Ideally, it could be shown by plotting the concentration of marker in a part of the gut at regular intervals or by examination of the decay curve of marker concentration after a single dose. Unfortunately, no methods are available to sample the contents of the lower intestine at regular intervals without seriously disturbing the physiology, and marker output in the stool gives too few and too irregular data for analysis on an individual basis.

Radiographs of the abdomen have been taken to demonstrate mixing of radio-opaque markers (Cassano et al., 1968) but the radiation dose involved precludes the use of this method for a detailed study of the mixing process.

We have demonstrated by the use of different radio-opaque markers that mixing occurs. Unequivocal evidence of this has been obtained in $34 \%$ of studies from the occurrence of mixed markers in the stool after different markers were given on successive days (Table). In addition, in $83 \%$ of the studies one or more stools contained all three markers, which in the presence of a daily bowel habit is strongly indicative of mixing. Counting the total numbers of marker in a stool is, however, a relatively insensitive method for showing mixing as it takes no account of the distribution of markers in individual stools. When stools are collected without distortion and examined it is evident that marker mixing occurs in most stools (Fig. 1). Better evidence is found when the overall picture of marker excretion in the stool is taken for a large group of studies (Fig. 2). This suggests that marker excretion follows an exponential pattern. Logarithmic conversion confirms this (Fig. 3).

These data suggest that in part of the gut a 'pool' of residue exists in which mixing occurs. This region is probably the caecum and right colon. Elliott and Barclay-Smith (1904) observed anti-peristalsis, retropulsion, and mixing of intestinal contents in the proximal colon of a number of animal species which had both carnivorous and herbivorous diets and suggested this might also occur in man. Halls (1965) observed similar occurrences in the right colon in man using radio-opaque markers and Edwards and Beck (1971) noted mixing of old and new effluent from the small intestine in the right colon.

From these observations it is possible to suggest the manner in which dietary residue passes through the gut. After a relatively rapid passage through the small intestine residue enters the caecum and right colon where it mixes with residue from previous meals. In this region a large and varied bacterial flora is active (Gorbach et al., 1967). The contents of this 'mixing pool' are gradually transferred to the left colon and sigmoid where further water and solutes are absorbed, thus increasing the viscosity and preventing further mixing. Storage occurs in the descending and the sigmoid colon and in the rectum. A similar view of the flow of colonic contents is arrived at by Christensen (1975) in a review of the myoelectric control of the colon in which he describes the coordinating mechanisms of colonic motility.

Transit through the large bowel thus has two components: time spent in the mixing region and time spent in sequential passage through and storage in the rest of the colon. The relative contribution of each of these to the overall colonic transit time probably varies considerably between individuals but some idea of a mean value may be obtained from the present data. If the caecum and the right colon are the mixing region then the first markers to leave it would do so soon after entering it. The time taken for $95 \%$ of the markers to leave the pool, assuming complete mixing, would be three times the turnover time for the pool (Zilversmit, 1960). The difference between the emergence in the stools of the first and last of the dose of marker would approximate to three times the turnover time. This difference is $\mathbf{5 9}$ hours and therefore the turnover time for the right colon pool would be 20 hours. Alternatively, if the delay in the emergence of the peak of markers is calculated (Fig. 2) the peak occurs at 0.7 of the transit time indicating that the proportion of total transit time (mean value 60 hours) associated with the mixing pool is 0.3 or 18 hours, which is in close agreement with the previous figure, although the calculations are substantially independent.

A practical consequence of this is that, if one wishes to have less than $5 \%$ of the residue of a previous dietary regime in stools collected in con- 
trolled dietary periods, the interval between starting a diet and starting a faecal collection should be twice the mean transit time.

\section{References}

Alvarez, W. C., and Freedlander, B. L. (1924). The rate of progress of food residues through the bowel. Journal of the American Medical Association, 83, 576-580.

Cassano, C. C., Vecchio, E. del, and Capurso, L. (1968). I tempi di transito gastro-intestinali. Radiologica Praktika, 18, 207-223.

Christensen, J. (1975). Myoelectric control of the colon. Gastroenterology, 68, 601-609.

Cummings, J. H., Jenkins, D. J. A., and Wiggins, H. S. (1976). Measurement of the mean transit time of dietary residue through the human gut. Gut, 17, 210-218.

Edwards, D. A. W., and Beck, E. R. (1971). Fecal flow, mixing and consistency. American Journal of Digestive Diseases, 16, 706-708.

Elliott, T. R., and Barclay-Smith, E. (1904). Antiperistalsis and other muscular activities of the colon. Journal of Physiology, 31, 272-304.

Findlay, J. M., Mitchell, W. D., Eastwood, M. A., Anderson, A. J. B., and Smith, A. N. (1974). Intestinal streaming patterns in cholerrhoeic enteropathy and diverticular disease. Gut, 15, 207-212

Gorbach, S. L., Plaut, A. S., Nahas, L., Weinstein, L., Spanknebel, G., and Levitan, R. (1967). Studies of intestinal microflora. 2 Microorganisms of the small intestine and their relations to oral and fecal flora. Gastroenterology, $53,856-867$.

Halls, J. (1965). Bowel content shift during normal defaecation. Proceedings of the Royal Society of Medicine, 58, 859-860.

Hill, M. J., Drasar, B. S., Aries, V., Crowther, J. S., Hawksworth, G., and Williams, R. E. O. (1971). Bacteria and aetiology of cancer of large bowel. Lancet, 1, 95-100.

Hoelzel, F. (1930). The rate of passage of inert materials through the digestive tract. American Journal of Physiology, 92, 466-497.

Zilversmit, D. B. (1960). The design and analysis of isotope experiments. American Journal of Medicine, 29, 832-848. 